\title{
Permanent correction of an inherited ectodermal dysplasia with recombinant EDA
}

\author{
Olivier Gaide \& Pascal Schneider \\ Institute of Biochemistry, BIL Biomedical Research Center, \\ University of Lausanne, CH-1066 Epalinges, Switzerland \\ Correspondence should be addressed to P.S.; e-mail: pascal.schneider@ib.unil.ch
}

Published online 7 April 2003; doi:10.1038/nm861

\begin{abstract}
X-linked hypohidrotic ectodermal dysplasia (XLHED; OMIM 305100) is a genetic disorder characterized by absence or deficient function of hair, teeth and sweat glands ${ }^{1}$. Affected children may experience life-threatening high fever resulting from reduced ability to sweat ${ }^{2}$. Mice with the Tabby phenotype share many symptoms with human XLHED patients because both phenotypes are caused by mutations of the syntenic ectodysplasin $A$ gene ( $E d a$ ) on the $\mathrm{X}$ chromosome $\mathrm{e}^{3,4}$. Two main splice variants of $E d a$, encoding EDA1 and EDA2, engage the tumor necrosis factor (TNF) family receptors EDAR and XEDAR, respectively ${ }^{5}$. The EDA1 protein, acting through EDAR, is essential for proper formation of skin appendages; the functions of EDA2 and XEDAR are not known. EDA1 must be proteolytically processed to a soluble form to be active ${ }^{6-9}$. Here, we show that treatment of pregnant Tabby mice with a recombinant form of EDA1, engineered to cross the placental barrier, permanently rescues the Tabby phenotype in the offspring. Notably, sweat glands can also be induced by EDA1 after birth. This is the first example of a developmental genetic defect that can be permanently corrected by short-term treatment with a recombinant protein.
\end{abstract}

Because the Tabby phenotype results from EDA deficiency and can be rescued by transgenic expression of $E d a 1 \mathrm{CDNA}^{10}$, we reasoned that administration of recombinant EDA to the developing embryo should cure Tabby mice and provide the basis for a possible treatment of XLHED. For this purpose, we generated recombinant proteins containing the receptor-binding domain of EDA fused to the $\mathrm{C}$ terminus of an IgG1 Fc domain (Fig. 1a). We called these recombinant fusion proteins Fc:EDA1 and Fc:EDA2. The Fc moiety ensures delivery of the protein from the maternal blood to the embryo through placental Fc receptors and also contributes to the stability of the molecule in vivo ${ }^{11,12}$. In addition, we expected the Fc moiety to substitute for the essential collagen domain of EDA that has been proposed to function as an aggregating domain ${ }^{7}$. Fusion of a dimeric Fc with a trimeric EDA was predicted to induce limited aggregation of the ligand ${ }^{13}$ (Fig. $1 b$ ). Fc:EDA1 and Fc:EDA2 were specific for their cognate receptors and did not bind the TNF-family receptor TROY, a close homolog of XEDAR that is also expressed in the $\operatorname{skin}^{14}$ (Fig. 1c).

Fc:EDA 1 was serially administered intravenously to pregnant Tabby mice at gestational days 11, 13 and 15 (400 $\mu \mathrm{g}$ per injection; referred to as the E11 protocol). Although we detected no change in the phenotype of treated mothers, reversion of the Tabby phenotype was readily apparent in their offspring. The benefits of Fc:EDA1 exposure during embryogenesis persisted throughout adulthood, and most of the characteristic Tabby fea- tures, including moderate runtism, reverted to a wild-type or wild-type-like phenotype (Fig. 2 and Table 1). The reappearance of tail hairs and their associated sebaceous glands, and of 'ear hairs' of the retro-auricular region, were particularly notable (Fig. $2 a$ and $b$ ). The density of hair follicles on the belly of 10day-old mice was markedly higher in treated mice than in wildtype controls, indicating ample dosage of recombinant Fc:EDA1 (Fig. $2 c$ ), although the density returned to normal in older animals. The coat of treated animals was often darker in color, probably because of an increased number of hair-associated melanocytes. The fur contained the long monotrich hairs, also called guard hairs, that are characteristic of wild-type mice (Fig. $2 c$ ). The tail lacked the characteristic 'kink' deformation at its extremity, and the jaw and molars of treated mice regained both their normal sizes and the classic wild-type pattern of sharp cusps (Fig. $2 b$ and $d$ ). In the Fc:EDA1-treated mice, the eye slit was normal and the eyelid contained fully developed meibomian glands, in contrast to untreated animals (Fig. $2 a$ and $e$ ). Meibomian glands secrete a thin film of lipids that prevents excessive dryness of the cornea. Consequently, the cornea of treated animals was devoid of the pathological keratinization often found in age-matched Tabby mice. The footpads of treated mice showed numerous dermal ridges and contained glandular tissues indistinguishable from wild-type sweat glands (Fig. 2f); sweat tests indicated that these glands were functional (Fig. $3 b$ ). Fc:EDA2 alone had no detectable effect on the reversion of the Tabby phenotype, indicating the specificity of Fc:EDA1.

Fc:EDA1 treatment was unable to correct at least two features of Tabby mice. Wild-type mice have an organized blend of four hair types (awl, auchene, zigzag and monotrich) that give fur a shiny and smooth texture, whereas the less organized fur of Tabby mice contains a single atypical hair type intermediate between awl and auchene (Fig. 2c). Fc:EDA1 treatment induced monotrich and some intermediate forms of hair but not the full spectrum of wild-type hairs such as zigzag (Fig. 2c). In addition, the small third molar that is missing in about $50 \%$ of Tabby mice bred from homozygous parents ${ }^{15}$ was also missing in five of ten lower-jaw quadrants of treated animals (Fig. 2d). Whereas Fc:EDA1 rescues tooth morphology but not hypodontia, transgenic expression of EDA1 corrects the number of molars but not their shape and size ${ }^{10}$. We wondered whether Fc:EDA2 would synergize with Fc:EDA1 in rescuing hypodontia and zigzag hairs. Preliminary experiments with combined injection of Fc:EDA1 and Fc:EDA2 did not rescue these features, suggesting that EDA2 may be irrelevant in this context or that the experimental conditions were inadequate for its action. Regulated expression of 
a

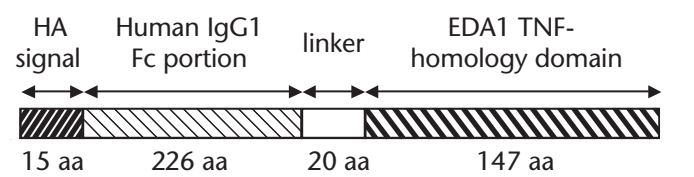

\section{$b$}

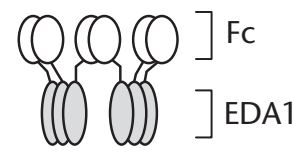

C

EDAR

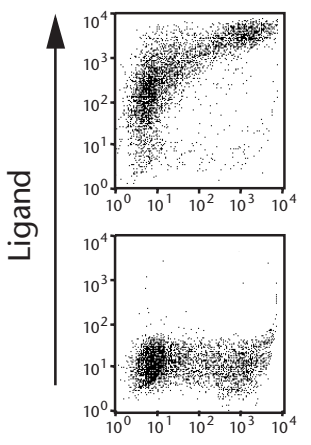

XEDAR
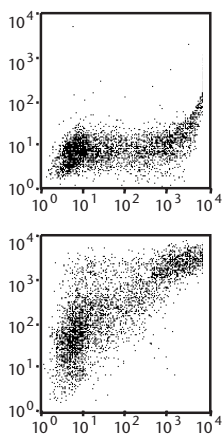

TROY
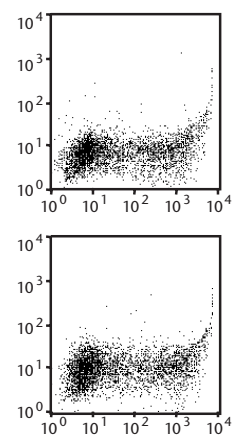

GFP
Mock
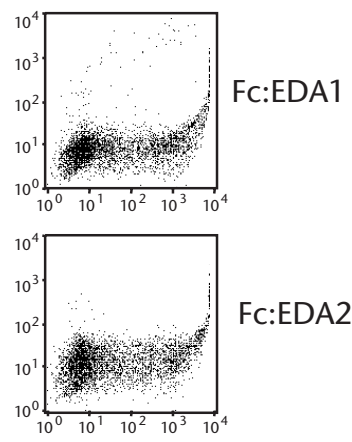

Fc:EDA2

Fig. 1 Features and receptor binding specificity of Fc:EDA1 and Fc:EDA2. $\boldsymbol{a}$, Scheme of the fusion protein Fc:EDA1. HA, hemagglutinin; aa, amino acids. $\boldsymbol{b}$, Putative organization of Fc:EDA1 as a hexamer. c, Flow cytometric analysis of 293T cells transfected with plasmids encoding the extracellular domains of the EDAR, XEDAR or TROY fused to a glycolipid anchor. Cells were analyzed for binding of Fc:EDA1 or Fc:EDA2 ( $y$ axis) and for expression of the co-transfection marker EGFP ( $x$ axis).

EDA1 and EDA2 (ref. 5) may result in gradients that cannot be reproduced by systemic administration of recombinant ligands, and truncation of EDA in the recombinant protein may affect some of its biological activity. Co-treatment resulted in a modest increase in the number of thin, kinked hairs (data not shown); this subtle phenotypic change may indicate cooperation of EDA2 with EDA1 in determining hair shape and is currently under investigation.

We next determined the time frame within which EDA1 can trigger differentiation of the various epidermal appendages. To this end, pregnant Tabby mice were serially injected intravenously with Fc:EDA1 at gestational days 15 and 17 (referred to as the E15 protocol). In separate experiments, newborn Tabby pups received a single intraperitoneal injection on either day 2 , 3,5 or 9 (referred to as protocols D2, D3, D5 and D9, respectively). Precursors of the different epithelial structures showed distinct capacities to respond to EDA1 (Fig. 3 and Table 1). The tooth phenotype was reversed by the E11 protocol, whereas the E15 protocol rescued the second molar only, consistent with its delayed development compared to the first molar ${ }^{16}$. Ear and monotrich hairs of the fur were restored by both the E11 and E15 protocols but not by the D2 protocol (Table 1), whereas tail hair was induced even when Fc:EDA1 treatment was initiated after birth (Fig. $3 c$ and $d$ ). Consistent with these results, Fc:EDA1 could be detected by immunohistochemistry in the skin $24 \mathrm{~h}$ after intraperitoneal administration (Fig. 3a). A dose-response experiment in 2-day-old newborn mice indicated that the minimal dose required for full reversion of the tail-hair phenotype was $1.5 \mathrm{mg} / \mathrm{kg}$, which was 30 times lower than in our standard protocol (data not shown). We also observed that hairs on the ventral face of the tail could be induced after a relatively late onset of treatment (D5 protocol), whereas those located on the dorsal, body-proximal part of the tail required the earlier D2 pro- tocol for induction (Fig. 3b). In postnatal treatment of Tabby mice, 13 days separated the onset of the treatment and the appearance of tail hair. This delay corresponds approximately to the time needed for hair formation in wild-type mice and Tabby mice treated in utero, both of which display tail hair on day 7 after birth. Numerous functional sweat glands were induced by the D3 and D5 protocols (Fig. 3b). Virtually none of the Tabby features was corrected by the D9 protocol, indicating that the triggering of epithelial structures by EDA1 is no longer possible at this stage.

Taken together, our results show that recombinant EDA1 can induce the formation of structures including guard, ear and tail hairs, sebaceous, sweat and meibomian glands, and normally shaped teeth. This is in agreement with previous genetic studies showing the involvement of dysfunctional EDA1 and EDAR in ectodermal dysplasia $^{7,10,17}$ and of the EDAR-associated death domain, TNF receptor-associated factor-6,

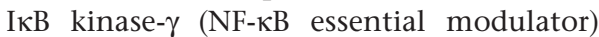
and NF- $\kappa \mathrm{B}$ intracellular signaling molecules acting downstream of $\mathrm{EDAR}^{18-21}$. In addition, we have shown that sustained action of EDA1 is not required for the maintenance of most, if not all, EDA1-dependent epidermal structures after an initial trigger. In particular, EDA seems not to be essential for the cycle of at least the monotrich and ear hairs, which is surprising given that EDA is expressed in hair follicles of adult skin ${ }^{22}$.

We conclude from our experiments that the Tabby syndrome, a developmental genetic disease, can be permanently cured by short-term treatment with a recombinant protein. The sequence of developmental events that allowed successful treatment of Tabby mice with Fc:EDA1 is essentially conserved in humans. Materno-fetal immunoglobulin transfer starts after six weeks of pregnancy and becomes maximal from week 12 onwards ${ }^{23}$, whereas hair development takes place between weeks 14 and 16 and sweat glands develop even later at week 20 (ref. 24). Similar approaches might be feasible for the treatment of other developmental diseases caused by deficient expression of a ligand. One

Table 1 Summary of the effects of Fc:EDA1 treatments

\begin{tabular}{lcccccc}
\hline Injection scheme & E11 & E15 & D2 & D3 & D5 & D9 \\
Pups/litters & $13 / 2$ & $5 / 1$ & $7 / 3$ & $4 / 2$ & $4 / 2$ & $2 / 2$ \\
Ear hair & ++ & ++ & - & - & - & - \\
Guard hair & ++ & ++ & - & - & - & - \\
Zigzag hair & - & - & - & - & - & - \\
Tail hair (ventral) & ++ & ++ & ++ & ++ & + & - \\
Tail hair (dorsal) & ++ & ++ & ++ & \pm & - & - \\
Tip of the tail & ++ & ++ & ++ & + & \pm & - \\
Molar shape & ++ & \pm & - & - & - & - \\
Eyelid & ++ & ++ & + & \pm & - & - \\
Meibomian glands & ++ & ++ & - & - & - & - \\
Sweat glands & ++ & ++ & ++ & ++ & ++ & \pm \\
Runtism & ++ & ++ & - & - & - & - \\
\hline
\end{tabular}

Treatments were initiated at the indicated days of embryonic (E) or postnatal (D) development. ++ , same as wild type; + , strongly reverted but not wild type; \pm , intermediate phenotype; -, same as Tabby. 
ARTICLES

$a$

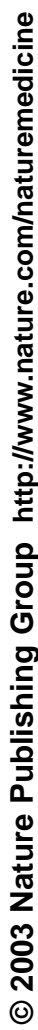

$b$

C
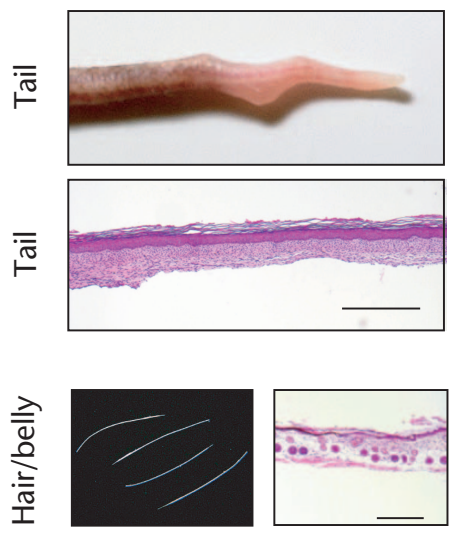

$d$
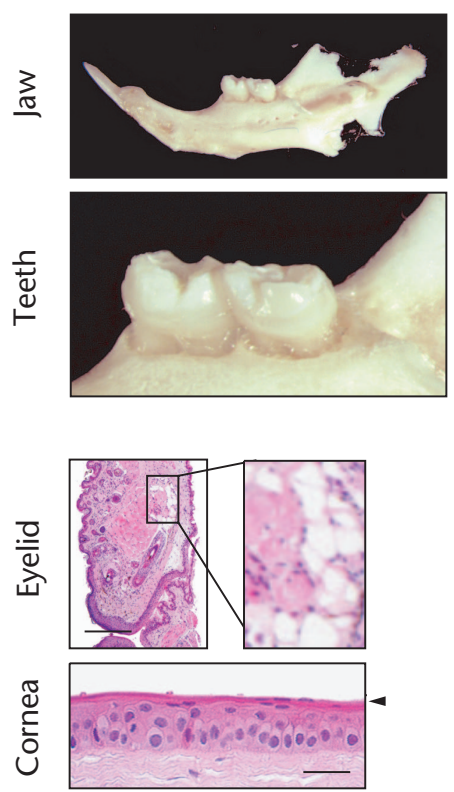

$f$
Tabby Fc:EDA1-treated
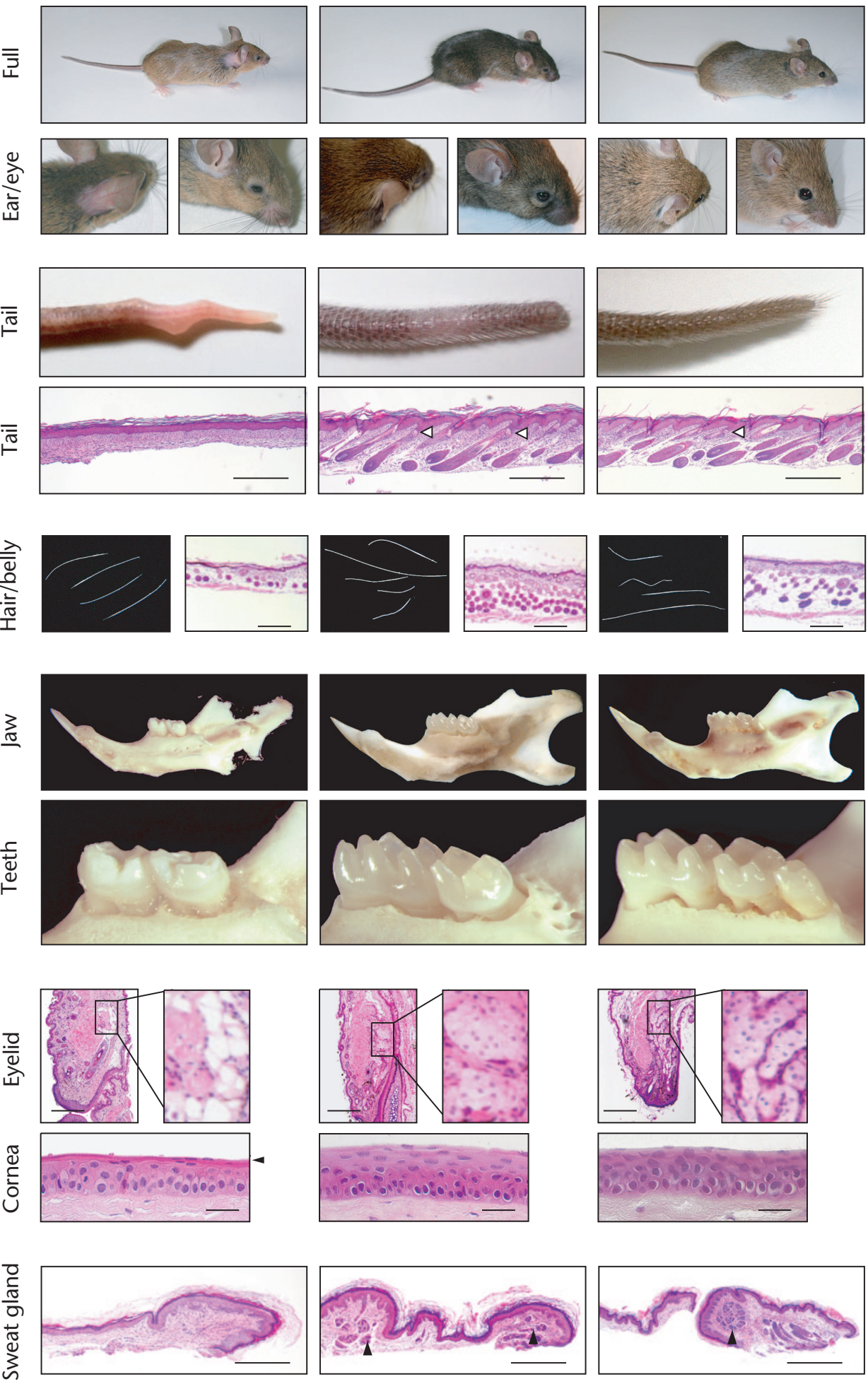

Fig. 2 In utero exposure to Fc:EDA1 results in reversion of the Tabby phenotype. Offspring of untreated Tabby mice or of Tabby mice treated with Fc:EDA1 are compared with offspring of wild-type mice in the same background. Adult mice and tissues are shown unless otherwise indicated. a, Entire animal and details of the retro-auricular and eye regions showing the darker fur coat, presence of ear hair and wide eye slit in treated Tabby mice. $\boldsymbol{b}$, Tip of the tail with reversal of tail baldness and kink phenotypes in treated mice. In the histological sections of tail skin, arrowheads point to sebaceous glands. Scale bar, $0.5 \mathrm{~mm}$. c, Hair types (left; adults) and belly skin section (right; 10-d-old mice). Scale bar, $0.5 \mathrm{~mm}$. In the wild-type mice, hair types are (from top to bottom) auchene, zigzag, awl and monotrich (guard). $\boldsymbol{d}$, Entire lower jaw (top) and detail of the molars (bottom). $\boldsymbol{e}$, Sections of the eyelid and enlargements of the indicated regions (top). Tabby mice lack meibomian glands, but these glands are present in wild-type and treated Tabby mice. Sections of the cornea showing keratinization (bottom; arrowhead) in age-matched Tabby mice. Scale bar, $0.25 \mathrm{~mm}$ (top) or $0.05 \mathrm{~mm}$ (bottom). $\boldsymbol{f}$, Footpad sections. Arrowheads indicate eccrine glands where present. In the mouse, sweat glands are located only in the footpads. Scale bar, $0.75 \mathrm{~mm}$. 
$a$
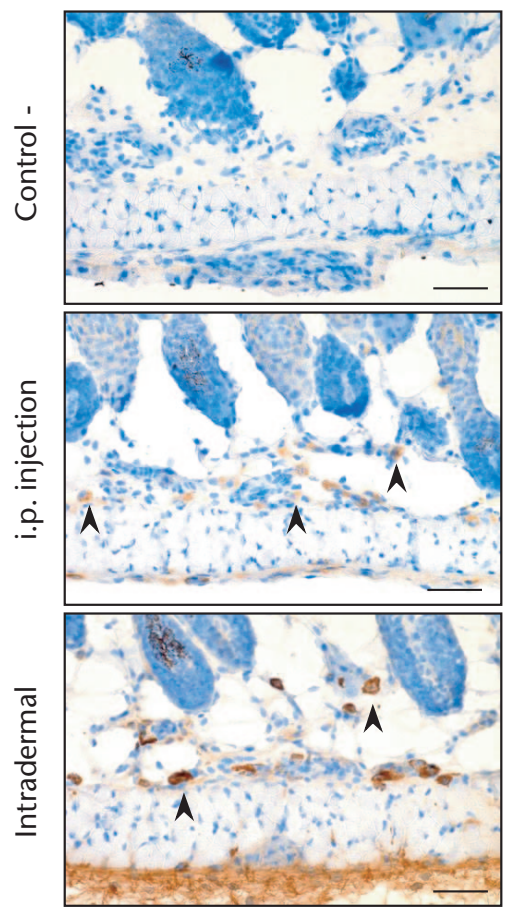

Fig. 3 Time-dependent restoration of sweat glands and tail hair in Fc:EDA1treated Tabby mice. $\boldsymbol{a}$, Basal skin section of a 3-d-old mouse injected intraperitoneally (i.p.) with Fc:EDA1 $24 \mathrm{~h}$ previously (center). Immunoreactivity (black arrows) with an antibody against human Fc indicates access of Fc:EDA1 to the skin. Top and bottom panels show negative (uninjected) and positive (intradermal injection of

Fc:EDA1 before tissue sectioning) controls, respectively. Scale bar, $0.1 \mathrm{~mm}$. b. Results of sweat tests done on Tabby mice exposed to Fc:EDA1 in utero (E11 and E15 protocols) or after birth (D2, D3, D5 and D9 protocols). An untreated Tabby mouse (Co-), a wild type mouse $(\mathrm{Wt} ; \mathrm{Co}+)$, and a Tabby mouse treated at day 2 with Fc:EDA2 are shown as controls. Sweat is detected as dark spots, mainly on the cushions and

C
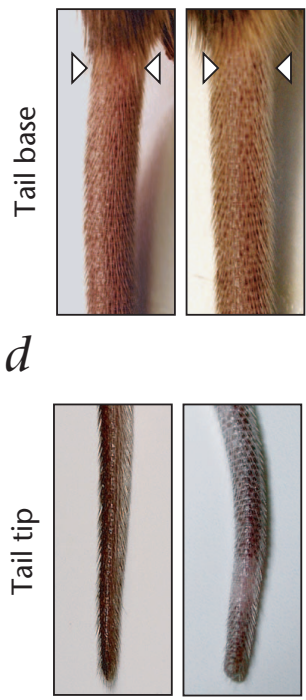

$b$
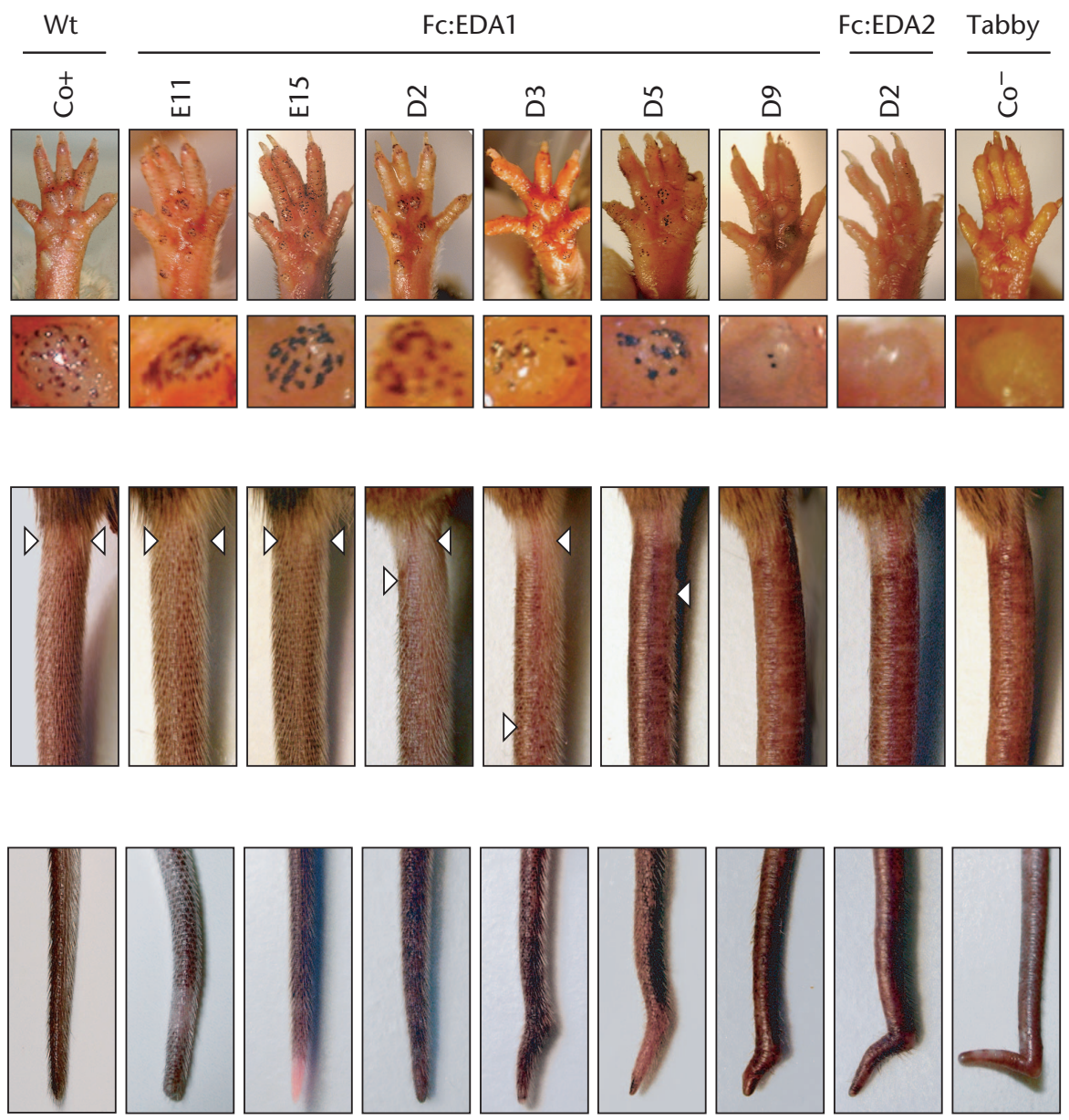

at the toetips. In Tabby mice treated at day 9, very few functional sweat glands can be detected. In each case, the detail of one cushion is also shown. c, Lateral view of the body-proximal part of the tail. The dorsal face is on the left. White arrows indicate the limit of tail hair growth, with hairs being on the distal part of the tail. $\boldsymbol{d}$, Effects of the different treatments on the kink at the tip of the tail. such example is acromesomelic chondrodysplasia and brachydactyly (OMIM 601146), which results from inactivating mutations in cartilage-derived morphogenetic protein-1 (CDMP1).

\section{Methods}

Recombinant fusion proteins. The Fc:EDA1 and Fc:EDA2 expression constructs in the PCR-3 vector (Invitrogen, NV Leek, The Netherlands) were constructed as described for Fc:FasL ${ }^{13}$. They encode the hemagglutinin signal peptide, the Fc portion of human IgG1 (amino acids 108-338 of GenBank accession number AAC82527, excluding the stop codon), a linker sequence (RSPQPQPKPQPKPEPEGSLQVD) and the receptor-binding domain of EDA1 (amino acids 245-391) or EDA2 (amino acids 245-389; Fig. $1 a$ ). This region of EDA is $100 \%$ conserved between mouse and human proteins. Chinese hamster ovary cells were transfected using Superfect (Qiagen, Basel, Switzerland) according to the manufacturer's protocol and selected $24 \mathrm{~h}$ later with $500 \mu \mathrm{g} / \mathrm{ml}$ of G418 (Invitrogen). Selected clones with the highest expression were inoculated at $10^{8}$ cells/liter in 2-liter roller bottles and grown for $14 \mathrm{~d}$ at $37^{\circ} \mathrm{C}$. Fc:EDA1 and Fc:EDA2 were purified on protein A-Sepharose columns as described, except that the elution buffer was $50 \mathrm{mM}$ citrate- $\mathrm{NaOH}(\mathrm{pH} 4.0)^{25}$. Proteins were concentrated to $2 \mathrm{mg} / \mathrm{ml}$ in PBS on an Ultrafree-15 centrifugal filter device (Millipore,
Bedford, Massachusetts) and stored at $-70{ }^{\circ} \mathrm{C}$. Yields were 6 and $3 \mathrm{mg}$ of protein per liter for Fc:EDA1 and Fc:EDA2, respectively. Endotoxin levels were below $0.1 \mathrm{ng}$ per $\mu \mathrm{g}$ protein. Both Fc:EDA1 and Fc:EDA2 displayed a half-life of $1.5 \mathrm{~d}$ in the circulation after intravenous administration (data not shown).

Binding studies. CDNA sequences encoding the extracellular domains of human EDAR (amino acids 1-183), human XEDAR (amino acids 1-123 preceded by an immunoglobulin signal peptide) and mouse TROY (amino acids 1-168) fused to the C-terminal, glycosyl phosphatidylinositolanchored portion of human TNF-related apoptosis-induced ligand receptor-3 (TRAILR3; amino acids 157-259) were cloned into the PCR-3 mammalian expression vector and co-transfected with an enhanced green fluorescent protein (EGFP) vector into 293T cells. Transfected cells were stained with Fc:EDA1 or Fc:EDA2 $(100 \mathrm{ng} / \mathrm{ml})$ followed by a phycoerythrincoupled goat antibody against human $\lg G$ and analyzed by 2-color flow cytometric analysis.

Treatment and analysis of Tabby mice. Mice were handled according to institutional and Swiss Federal Veterinary Office guidelines. Homozygous female and hemizygous male Tabby mice (000314; Jackson Laboratory, Bar 
Harbor, Maine) were mated for 3 consecutive nights. Pregnant females received serial intravenous injections of $400 \mu \mathrm{g}$ of Fc:EDA1 or Fc:EDA2 at $2 \mathrm{mg} / \mathrm{ml}$ in PBS at days 11,13 and 15 (protocol E11) or days 15 and 17 (protocol E15) after conception. Protocol E15 was also used for co-injection of $400 \mu \mathrm{g}$ each of Fc:EDA1 and Fc:EDA2. Timing of the injection was deduced by the day of birth of the pups, the length of the pregnancy being $19 \mathrm{~d}$ for this strain in our laboratory. For post-birth treatments, newborn pups received a single intraperitoneal injection of either Fc:EDA1 or Fc:EDA2 at day $2(40 \mu \mathrm{g}), 3(40 \mu \mathrm{g}), 5(60 \mu \mathrm{g})$ or $9(100 \mu \mathrm{g})$. Dose-response experiments with Fc:EDA1 $(40,14,4,1.5$ or $0.5 \mu \mathrm{g})$ were done on 2-d-old littermates in 3 independent experiments with identical results. Adult or 10-d-old mice were killed and skin samples of the tail, footpad, eyelid, cornea and belly were fixed for $24 \mathrm{~h}$ in $4 \%$ formaldehyde in PBS. Samples were dehydrated, paraffin-embedded, sectioned $(10 \mu \mathrm{m})$ and stained with $\mathrm{H \& E}$. Back skin of a mouse injected intraperitoneally at day 3 with $40 \mu \mathrm{g}$ of Fc:EDA1 was subjected $24 \mathrm{~h}$ later to frozen-section immunohistochemistry using peroxidase-coupled donkey antibody against human IgG (1:100 dilution; Jackson Immunoresearch, Milan Analytica, La Roche, Switzerland). Back skin from control (non-injected) pups was infiltrated with $2 \mathrm{mg} / \mathrm{ml} \mathrm{Fc:EDA} 1$ or left untreated to serve as positive or negative controls, respectively.

Jaws of adult mice were cleaned surgically from attached soft tissue and boiled for $1 \mathrm{~h}$ in PBS. The remaining soft-tissue debris was removed either manually or by a 1 -h incubation at $37{ }^{\circ} \mathrm{C}$ with $150 \mu \mathrm{g} / \mathrm{ml}$ of proteinase $\mathrm{K}$ (Roche, Rotkreuz, Switzerland).

Sweat tests. Hind paws of immobilized animals were painted with a solution of $3 \%(\mathrm{wt} / \mathrm{vol})$ iodine in ethanol. Once dry, the paws were painted with a suspension of $40 \%(\mathrm{wt} / \mathrm{vol})$ starch in mineral oil. Pictures were taken 1 min later; sweat was detected as dark spots. Mice testing negative continued to do so even after further heat treatment for 1 min under an infrared lamp.

\section{Acknowledgments}

We thank J. Tschopp for support; E. Säuberli and G. Badic for assistance with histology; and H. Everett, M. Mikkola and J. Zonana for helpful comments. This work was supported by grants from the Swiss National Science Foundation and the National Center of Competence in Research.

\section{Competing interests statement}

The authors declare competing financial interests: see the Nature Medicine website for details.

\section{RECEIVED 11 NOVEMBER 2002; ACCEPTED 14 MARCH 2003}

1. Pinheiro, M. \& Freire-Maia, N. Ectodermal dysplasias: a clinical classification and a causal review. Am. J. Med. Genet. 53, 153-162 (1994).

2. Clarke, A., Phillips, D.I., Brown, R. \& Harper, P.S. Clinical aspects of X-linked hy- pohidrotic ectodermal dysplasia. Arch. Dis. Child. 62, 989-996 (1987)

3. Monreal, A.W., Zonana, J. \& Ferguson, B. Identification of a new splice form of the EDA1 gene permits detection of nearly all X-linked hypohidrotic ectodermal dysplasia mutations. Am. J. Hum. Genet. 63, 380-389 (1998).

4. Srivastava, A.K. et al. The Tabby phenotype is caused by mutation in a mouse homologue of the EDA gene that reveals novel mouse and human exons and encodes a protein (ectodysplasin-A) with collagenous domains. Proc. Natl. Acad. Sci. USA 94, 13069-13074 (1997).

5. Yan, M. et al. Two-amino acid molecular switch in an epithelial morphogen that regulates binding to two distinct receptors. Science 290, 523-527 (2000).

6. Laurikkala, J. et al. Regulation of hair follicle development by the TNF signal ectodysplasin and its receptor Edar. Development 129, 2541-2553 (2002).

7. Schneider, P. et al. Mutations leading to X-linked hypohidrotic ectodermal dysplasia affect three major functional domains in the tumor necrosis factor family member ectodysplasin-A. J. Biol. Chem. 276, 18819-18827 (2001).

8. Tucker, A.S. et al. Edar/Eda interactions regulate enamel knot formation in tooth morphogenesis. Development 127, 4691-4700 (2000).

9. Thesleff, I. \& Mikkola, M.L. Death receptor signaling giving life to ectodermal organs. Science STKE 2002, PE22 (2002).

10. Srivastava, A.K. et al. Ectodysplasin-A1 is sufficient to rescue both hair growth and sweat glands in Tabby mice. Hum. Mol. Genet. 10, 2973-2981 (2001).

11. Rennert, P.D., Browning, J.L. \& Hochman, P.S. Selective disruption of lymphotoxin ligands reveals a novel set of mucosal lymph nodes and unique effects on lymph node cellular organization. Int. Immunol. 9, 1627-1639 (1997).

12. Colcher, D. et al. Pharmacokinetics and biodistribution of genetically-engineered antibodies. Q. J. Nucl. Med. 42, 225-241 (1998).

13. Holler, N. et al. Two adjacent trimeric Fas ligands are required for Fas signaling and formation of a death-inducing signaling complex. Mol. Cell. Biol. 23, $1428-1440$ (2002)

14. Kojima, T. et al. TROY, a newly identified member of the tumor necrosis factor receptor superfamily, exhibits a homology with Edar and is expressed in embryonic skin and hair follicles. J. Biol. Chem. 275, 20742-20747 (2000).

15. Kristenova-Cermakova, P., Peterka, M., Lisi, S., Lesot, H. \& Peterkova, R. Postnatal lower jaw dentition in different phenotypes of tabby mice. Connect. Tissue Res. 43, 283-288 (2002).

16. Lesot, H. et al. Mouse molar morphogenesis revisited by three-dimensional reconstruction. II. Spatial distribution of mitoses and apoptosis in cap to bell staged first and second upper molar teeth. Int. J. Dev. Biol. 40, 1017-1031 (1996).

17. Monreal, A.W. et al. Mutations in the human homologue of mouse $d l$ cause autosomal recessive and dominant hypohidrotic ectodermal dysplasia. Nat. Genet. 22, 366-369 (1999).

18. Naito, A. et al. TRAF6-deficient mice display hypohidrotic ectodermal dysplasia. Proc. Natl. Acad. Sci. USA 99, 8766-8771 (2002).

19. Headon, D.J. et al. Gene defect in ectodermal dysplasia implicates a death domain adapter in development. Nature 414, 913-916 (2001).

20. Schmidt-Ullrich, R. et al. Requirement of NF-KB/Rel for the development of hair follicles and other epidermal appendices. Development 128, 3843-3853 (2001).

21. Doffinger, R. et al. X-linked anhidrotic ectodermal dysplasia with immunodeficiency is caused by impaired NF-KB signaling. Nat. Genet. 27, 277-285 (2001).

22. Montonen, O. et al. The gene defective in anhidrotic ectodermal dysplasia is expressed in the developing epithelium, neuroectoderm, thymus, and bone. J. Histochem. Cytochem. 46, 281-289 (1998).

23. Jauniaux, E. et al. Materno-fetal immunoglobulin transfer and passive immunity during the first trimester of human pregnancy. Hum. Reprod. 10, 3297-3300 (1995).

24. Ersch, J. \& Stallmach, T. Assessing gestational age from histology of fetal skin: an autopsy study of 379 fetuses. Obstet. Gynecol. 94, 753-757 (1999).

25. Schneider, P. Production of recombinant TRAIL and TRAIL receptor: Fc chimeric proteins. Meth. Enzymol. 322, 325-345 (2000). 